\title{
СИСТЕМА АВТОМАТИЧЕСКОГО РЕГУЛИРОВАНИЯ ТЕМПЕРАТУРЫ НЕСТАЦИОНАРНОГО ОБЪЕКТА
}

\author{
Павлов А.И., \\ Одесская национальная академия пищевых технологий, г. Одесса
}

Copyright (C) 2014 by author and the journal "Automation technological and business - processes". This work is licensed under the Creative Commons Attribution International License (CC BY). http://creativecommons.org/licenses/by/4.0/

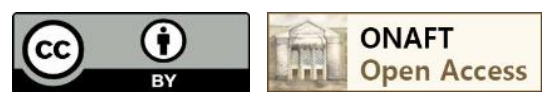

DOI: $10.15673 / 2312-3125.21 /$

\section{Аннотация}

Рассмотрен способ динамической коррекции сигнала управления автоматического регулятора. Способ основан на измерении величины теплового потока нелинейного диссипативного объекта. Тепловой поток интерпретируется как «внутреннее» управляющее воздействие объекта регулирования. Сигнал управления корректируется по изменению величины теплового потока объекта. Алгоритм регулирования реализуется искусственной нейронной сетью простейшей структуры. Число входных сигналов нейронной сети равно четырем: задание, динамическая ошибка, тепловой поток, внешнее возмущение. Функция активации выхода нейронной сети - линейная с ограничениями. Такая система автоматического регулирования обеспечивает высокое качество переходных процессов в ней при болыших изменениях параметров объекта. Внешние возмущения и изменения задания также слабо влияют на динамику системы. Определены границы области нормальных режимов работы системы регулирования. Представлены результаты исследований на имитационной модели системы автоматического регулирования.

\section{Abstract}

The method of dynamic correction of signal of management of automatic regulator is considered. A method is based on measuring of size of thermal stream of nonlinear dissipative object. The thermal stream is interpreted as «internal» managing influence of adjusting object. A management signal is corrected on the change of size of thermal stream of object. An adjusting algorithm will be realized by the artificial neural network of the simplest structure. The number of input signals of the neural network is equal to four: task, dynamic error, thermal stream, external disturbances. Function of activating of exit of neural network - liner with limitations. Such structure of the automatic control system provides high quality of transients in her at the large changes of parameters of object. External indignations and changes of task also poorly influence on the dynamics of the adjusting system. The borders of area of the normal modes of operations of the automatic control system are certain. The researches results on the simulation model of the automatic control system are presented.

Ключевые слова

Объект, регулятор, модель, система.

\section{Введение}

В [1] был расмотрен простой способ динамической коррекции коэффициента передачи пропорциональноинтегрального автоматического регулятора вследствие изменения в широком диапазоне коэффициента передачи объекта. Использование этого способа требует непрерывного вычисления текущего значения коэффициента 


\section{1 ПИТАННЯ ТЕОРІЇ, МЕТОДИ ТА АЛГОРИТМИ ЕФЕКТИВНОГО АВТОМАТИЧНОГО УПРАВЛІННЯ ОБ’ЄКТАМИ ХІМІКО-ТЕХНОЛОГІЧНОГО ТИПУ}

передачи объекта регулирования.

В [2] показано, что для компенсации изменения величины коэффициента передачи объекта требуется корректирующая цепь, содержащая два динамических звена: интегро-дифференцирующее и апериодическое первого порядка. И, значит, возникает необходимость определения (оптимизации) параметров указанных динамических звеньев.

Все публикации (а их великое множество), относящиеся к проблеме компенсации управляющего воздействия регулятора вследствие изменения коэффициента передачи объекта, свидетельствуют о необходимости построения корректирующих цепей. Действительно, иного пути нет, если используются линейные алгоритмы регулирования. Все более возрастающая сложность предлагаемых структур управляющей части системы (то есть алгоритмов, реализуемых ею) привела к тупиковой ситуации: размерность адаптивных регуляторов во много раз превышает размерность используемых имитационных моделей объектов. Выйти за пределы этого порочного круга можно, решая двуединую задачу: повышать адекватность моделей объектов и одновременно упрощать структуру управляющей части системы. Что касается первой задачи, предложения были высказаны ранее [3]. Более тщательное и углубленное рассмотрение физического процесса, протекающего в реальном объекте регулирования, обычно позволяет выявить в нем «внутреннее» управляющее воздействие. Такой подход к построению имитационной модели объекта регулирования обеспечивает возможность существенного упрощения структуры управляющей подсистемы [1].

\section{Основная часть}

Разработка имитационной модели системы автоматического регулирования основывалась на двух принципах системного анализа: принципе полноты и принципе простоты.

Первый из них использовался для построения имитационной модели объекта регулирования, что привело к повышению ее адекватности и, кроме того, позволило выявить промежуточную координату - тепловой поток как «внутреннее» управляющее воздействие [3].

Использование второго принципа обеспечивает предельную простоту управляющей подсистемы. Отказ от классических линейных алгоритмов регулирования позволяет радикально упростить структуру управляющей подсистемы. Однако их отрицание отнюдь не является полным: использование динамических звеньев в структуре регулятора, и в первую очередь интегратора, необходимо.

Такой (синергетический) подход приводит к двухконтурной системе регулирования. При этом динамические свойства внутреннего контура системы управления зависят только от вида исполнительного механизма (ИМ), который определяется проектировщиком системы регулирования посредством имитационного моделирования, то есть носят, в определенной мере, субъективный характер. Если предполагается использование пневматического мембранного ИМ, оснащенного позиционером, то упрощенная математическая модель (передаточная функция ИМ) имеет вид:

$$
\mathrm{W}_{\text {им }}(\mathrm{P})=\frac{\mathrm{e}^{-\tau_{1} \mathrm{P}}}{\left(\mathrm{T}_{1} \mathrm{P}+1\right)^{2}} .
$$

Численные величины параметров $\tau_{1}$ и $\mathrm{T}_{1}$ зависят от типоразмера ИМ и в процессе его работы остаются (при надлежащем уходе за исполнительным устройством) практически постоянными.

Структурная схема имитационной модели системы регулирования в этом случае будет иметь вид, показанный на рис. 1.

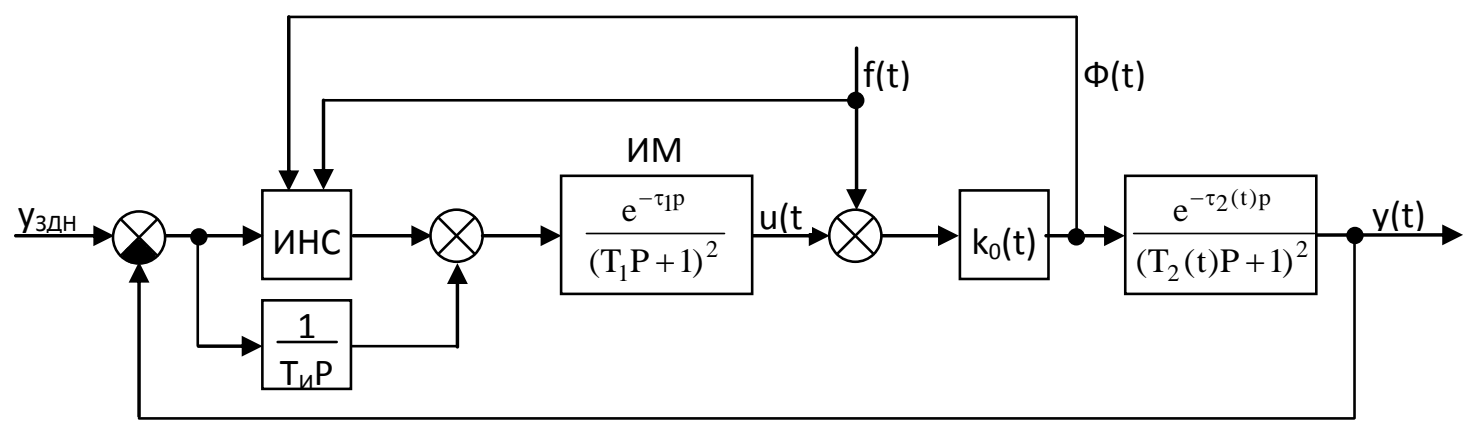

Рис. 1 - Структурная схема стабилизирующей системы автоматического регулирования 


\section{1 ПИТАННЯ ТЕОРІї, МЕТОДИ ТА АЛГОРИТМИ ЕФЕКТИВНОГО АВТОМАТИЧНОГО} УПРАВЛІННЯ ОБ'ЄКТАМИ ХІМІКО-ТЕХНОЛОГІЧНОГО ТИПУ

$\mathrm{y}_{\text {здн }}, \mathrm{y}(\mathrm{t})$ - соответственно заданное и фактическое значения регулируемой координаты;

ИНС - искусственная нейронная сеть; $\mathrm{u}(\mathrm{t})$ - управляющее воздействие;

$\mathrm{f}(\mathrm{t})$ - координатное возмущение по каналу управления; $\Phi(\mathrm{t})$ - величина теплового потока

Таким образом, передаточная функция первого участка объекта имеет вид:

$$
\mathrm{W}_{1}(\mathrm{P})=\frac{\mathrm{k}_{0}(\mathrm{t}) \mathrm{e}^{-\tau_{1} \mathrm{p}}}{\left(\mathrm{T}_{1} \mathrm{P}+1\right)^{2}},
$$

причем при работе реального объекта $\mathrm{k}_{0}$ уменьшается (может снижаться в 2 и более раз), а величины $\tau_{1}, \mathrm{~T}_{1}$ и их соотношение $\tau_{1} / T_{1}$ при этом остаются постоянными.

Если же разработчик системы регулирования выбирает электрический ИМ пропорционального действия, то его структурная схема имеет вид, показанный на рис. 2.

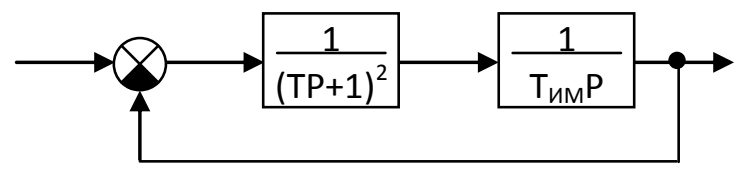

Рис. 2 - Структурная схема электрического ИМ пропорционального действия

Величина постоянной времени Т зависит от типоразмера ИМ, а оптимальная величина $\mathrm{T}_{\text {им }}$ определяется посредством имитационного моделирования системы регулирования [4].

Динамические свойства по каналу управления второго участка объекта регулирования соответствуют, например, передаточной функции

$$
\mathrm{W}_{2}(\mathrm{P})=\frac{\mathrm{e}^{-\tau_{2}(\mathrm{t}) \mathrm{p}}}{\left(\mathrm{T}_{2}(\mathrm{t}) \mathrm{P}+1\right)^{2}},
$$

причем в процессе работы технологического агрегата (аппарата либо машины) величины $\tau_{2}$ и $\mathrm{T}_{2}$ обычно (по известным причинам) увеличиваются; меняется (в сторону возрастания) и их соотношение $\tau_{2} / \mathrm{T}_{2}$.

Считая задачу упрощения структуры управляющей подсистемы весьма актуальной, в соответствие с [5] использован вариант формирования сигнала управления одним искусственным нейроном (обучение становится настолько простым, что отпадает необходимость (при небольшом количестве входных сигналов) применять программу оптимизации величин коэффициентов синапсов). На входы нейрона подаются всего четыре сигнала: задание, динамическая ошибка, величина теплового потока объекта и координатное возмущение (отклонение величины давления пара от номинальной величины). Активационная функция - линейная с ограничениями.

Имитационное моделирование проводилось с использованием системы программирования KONGRAF.

В варианте использования пневматического ИМ приняты значения: $\tau_{1}=0,3$ с, $\mathrm{T}_{1}=1,5$ с; в варианте использоания электрического ИМ приняты значения: $\mathrm{T}=0,2 \mathrm{c}, \mathrm{T}_{\text {им }}=10 \mathrm{c}$. Постоянная времени интегратора $\mathrm{T}_{\text {И }}=$ 12 с и в процессе моделирования не менялась. Непрерывное координатное возмущение - сумма трех синусоидальных сигналов с параметрами: $\mathrm{A}_{1}=8 \%, \mathrm{~F}_{1}=0,005$ pad $/ \mathrm{c} ; \mathrm{A}_{2}=2 \%, \mathrm{~F}_{2}=0,01$ pad $/ c ; \mathrm{A}_{3}=5 \%, \mathrm{~F}_{3}=0,001$ $\mathrm{pad} / \mathrm{c}$.

При скачкообразных изменениях задания на \pm 20 \% переходные процессы апериодические; при скачках координатного возмущения - колебательные быстро затухающие.

На рис. 3 представлена область нормальных режимов (ОНР) работы системы регулирования. Так, например, для всех точек на отрезке АВ максимальная динамическая ошибка (по модулю) не превышала 0,8 \% при изменении $0,6 \leq \mathrm{K}_{0} \leq 1,6$. Минимальная величина $\mathrm{K}_{0}=0,4$ (при меньших значениях $\mathrm{K}_{0}$ регулирующий орган выходит на отметку $100 \%$, и система регулирования утрачивает работоспособность); при этом динамическая ошибка увеличивается до 4 \%: начинает сильно влиять нелинейность расходной характеристики регулирующего органа (использовалась равнопроцентная пропускная характеристика). 


\section{1 ПИТАННЯ ТЕОРІЇ, МЕТОДИ ТА АЛГОРИТМИ ЕФЕКТИВНОГО АВТОМАТИЧНОГО УПРАВЛІННЯ ОБ'ЄКТАМИ ХІМІКО-ТЕХНОЛОГІЧНОГО ТИПУ}

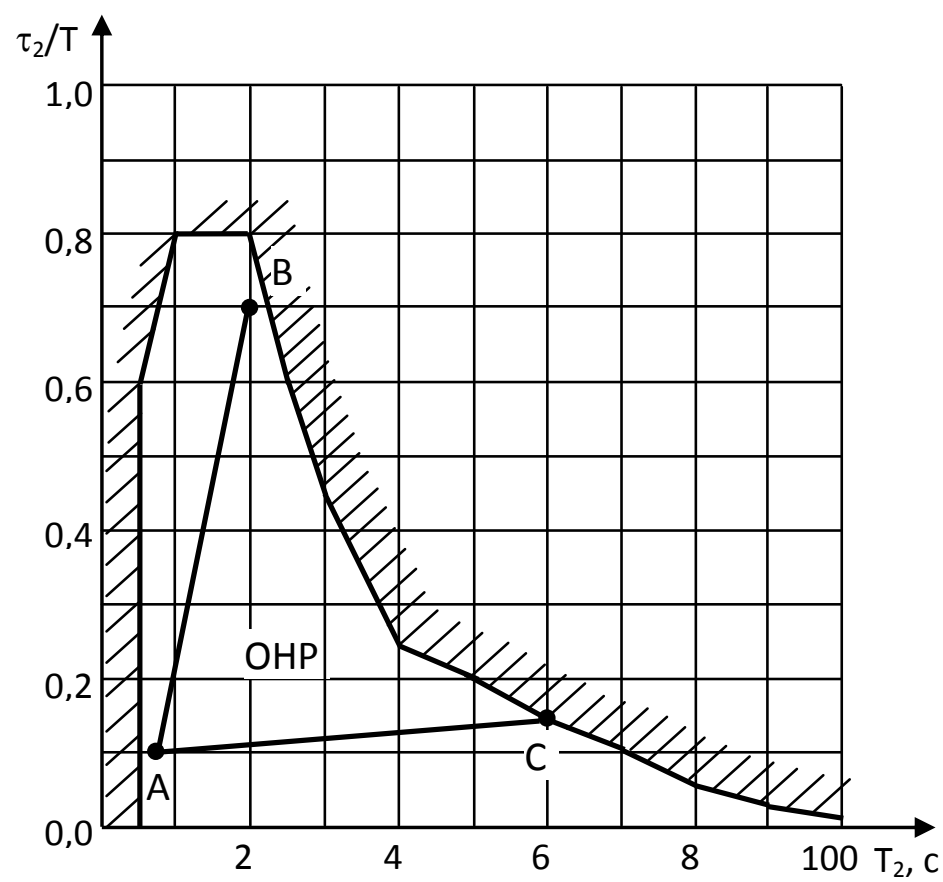

Рис. 3 - Область нормальных режимов работы системы автоматического регулирования

Если отрезок $\mathrm{AB}$ в ОНР характеризует опережающий рост $\tau_{2}$ по сравнению с увеличением $\mathrm{T}_{2}$, то отрезок $\mathrm{AC}$ напротив отражает опережающий рост $\mathrm{T}_{2}$ по сравнению с увеличением $\tau_{2}$. Но и для прямой $\mathrm{AC}$ характерна такая же величина динамической ошибки. По всему пространству ОНР, если $0,6 \leq \mathrm{K}_{0} \leq 1,6$, то максимальная динамическая ошибка гарантированно не превысит порога в $1 \%$.

\section{Выводы}

В случаях больших параметрических возмущений объекта применение в структуре регулятора искусственной нейронной сети (даже в варианте использования всего одного нейрона) позволяет избавиться от необходимости разработки корректирующих цепей, при условии, что выявлено «внутреннее» управляющее воздействие объекта (в данном случае - величина теплового потока).

Использование информации об изменении «внутреннего» управляющего воздействия объекта приводит к двухконтурной («каскадной») структуре системы автоматического регулирования; при этом динамические свойства первого участка объекта регулирования определяются видом и техническими (паспортными) характеристиками исполнительного механизма.

Система регулирования обладает хорошими демпфирующими способностями при мгновенных изменениях возмущения объекта по каналу управления и задания.

Имитационное моделирование позволило определить область нормальных режимов работы системы регулирования, в пределах которой она обладает выраженными адаптивными свойствами.

\section{Литература}

1. Павлов А. И. Динамическая коррекция коэффициента передачи пропорционально-интегрального регулятора [Текст] / А. И. Павлов // Автоматизация технологических и бизнес-процессов. - Одесса : ОНАПТ, 2015. - № 1. - Т. 7. - C. 31-34;

2. Водічев В. А. Дослідження динамічних характеристик параметричних систем керування технологічними процессами [Текст] / В. А. Водічев, П. М. Монтік, Алі Алдаїрі // Автоматизация технологических и бизнеспроцессов. - Одесса : ОНАПТ, 2014. - № 3 (19). - С. 4-7;

3. Павлов А. И. Повышение адекватности имитационных моделей нелинейных диссипативных объектов [Текст] / А. И. Павлов // Автоматизация технологических и бизнес-процессов. - Одесса : ОНАПТ, 2014. № 17. - C. 4-7; 


\title{
1 ПИТАННЯ ТЕОРЇ̈, МЕТОДИ ТА АЛГОРИТМИ ЕФЕКТИВНОГО АВТОМАТИЧНОГО УПРАВЛІННЯ ОБ’ЄКТАМИ ХІМІКО-ТЕХНОЛОГІЧНОГО ТИПУ
}

4. Павлов А. И. Виртуальный стенд для определения оптимального по быстродействию исполнительного устройства [Текст] / А. И. Павлов // Автоматизация технологических и бизнес-процессов. - Одесса : ОНАПТ, 2014. - № 4. - Т. 6. - С. 130-137;

5. Павлов А. И. Технология проектирования нейронных регуляторов [Текст] / А. И. Павлов // Автоматизация технологических и бизнес-процессов. - Одесса : ОНАПТ, 2010. - № 4. - С. 15-20;

6. Павлов А. И. Повышение эффективности управляющих воздействий автоматических регуляторов [Текст] / А. И. Павлов // Автоматизация технологических и бизнес-процессов. - Одесса : ОНАПТ, 2011. - № 5, 6. C. 31-37.

References

1. Pavlov A. I. Dinamicheskaya korrektsiya koeffitsienta peredachi proportsional'no-integral'nogo regulyatora [Tekst] / A. I. Pavlov // Avtomatizatsiya tekhnologicheskikh i biznes-protsessov. - Odessa : ONAPT, 2015. - № 1. - T. 7. - S. 31-34.;

2. Vodichev V. A. Doslidzhennya dynamichnykh kharakterystyk parametrychnykh system keruvannya tekhnologichnymy protsessamy [Tekst] / V. A. Vodichev, P. M. Montik, Ali Aldayiri // Avtomatizatsiya tekhnologicheskikh i biznes-protsessov. - Odessa: ONAPT, 2014. - № 3 (19). - S. 4-7.;

3. Pavlov A. I. Povysheniye adekvatnosti imitatsionnykh modeley nelineynykh dissipativnykh ob'ektov [Tekst] / A. I. Pavlov // Avtomatizatsiya tekhnologicheskikh i biznes-protsessov. - Odessa: ONAPT, 2014. - № 17. - S. 4-7.;

4. Pavlov A. I. Virtual'nyy stend dlya opredeleniya optimal'nogo po bystrodeystviyu ispolnitel'nogo ustroystva [Tekst] / A. I. Pavlov // Avtomatizatsiya tekhnologicheskikh i biznes-protsessov. - Odessa: ONAPT, 2014. - № 4. - T. 6. - S. 130-137.;

5. Pavlov A. I. Tekhnologiya proektirovaniya neyronnykh regulyatorov [Tekst] / A. I. Pavlov // Avtomatizatsiya tekhnologicheskikh i biznes-protsessov. - Odessa: ONAPT, 2010. - № 4. - S. 15-20.;

6. Pavlov A. I. Povysheniye effektivnosti upravlyayuschikh vozdeystviy avtomaticheskikh regulyatorov [Tekst] / A. I. Pavlov // Avtomatizatsiya tekhnologicheskikh i biznes-protsessov.- Odessa: ONAPT, 2011.- № 5, 6. -S. 31-37.

\section{МОДЕЛЬ АВТОМАТИЗОВАНОЇ СИСТЕМИ МОНІТОРИНГУ СТАНУ ІРУНТІВ СІЛЬСЬКОГОСПОДАРСЬКОГО ПІДПРИЕМСТВА}

\author{
Молодецька К. В., Нетребко Р. В. \\ Житомирський національний агроекологічний університет \\ Житомирський військовий інститут ім. С. П. Корольова \\ Copyright (C) 2014 by author and the journal “Automation technological and business - processes". \\ This work is licensed under the Creative Commons Attribution International License (CC BY). \\ http://creativecommons.org/licenses/by/4.0/
}

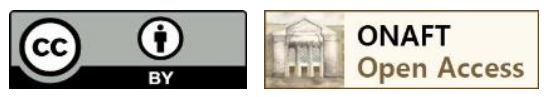

DOI: $10.15673 / 2312-3125.21 /$

\section{Анотація}

Відсутність ефективних систем автоматизації процесів оперативного моніторингу стану грунтів с основною причиною зниження якості прогнозу і врожайності, ускладнення процесів розробки агротехнічних планів 